\title{
Unusual cause of superior vena cava syndrome diagnosed with transesophageal echocardiography
}

\author{
[Un cas inhabituel de syndrome de la veine cave supérieure dépisté grâce à
}

l'échocardiographie transasophagienne]

Atilio Barbeito MD, ${ }^{*}$ Shahar Bar-Yosef MD, ${ }^{*}$ James E. Lowe MD, $†$ Broadus Z. Atkins MD, $†$ Jonathan B. Mark MD*

Purpose: An unusual case of superior vena cava (SVC) syndrome caused by an infected right atrial-SVC junction thrombus may be diagnosed using transesophageal echocardiography.

Clinical features: A 59-yr-old male with end-stage renal disease requiring hemodialysis presented with fungemia and later developed facial and bilateral upper extremity edema. Transesophageal echocardiography revealed subtotal occlusion of the SVC at its junction with the right atrium. The mass was surgically removed with cardiopulmonary bypass support. Pathological examination of the mass confirmed the presence of a large fungal colony of Candida species mixed in the thrombus. The patient's signs and symptoms of SVC obstruction resolved, and he was discharged from the hospital four weeks later in stable condition.

Conclusion: Although usually caused by extrinsic tumour compression, SVC syndrome can result from intravascular caval obstruction. This etiology should also be considered in the differential diagnosis, particularly in patients with intravascular devices. Transesophageal echocardiography is a valuable diagnostic tool in these cases.

CAN J ANESTH 2008/ 55: $11 /$ pp 774-778

Objectif: Un cas inhabituel de syndrome de la veine cave supérieure provoqué par un thrombus infecté à la jonction de l'oreillette droite et de la veine cave supérieure peut être dépisté grâce à l'échocardiographie transœsophagienne.

Éléments cliniques : Un homme de 59 ans souffrant d'insuffisance rénale terminale et nécessitant une hémodialyse a manifesté une septicémie à champignons, puis un œdème facial et bilatéral des membres supérieurs. L'échocardiographie transœsophagienne a révélé une occlusion sous-totale de la veine cave supérieure à sa jonction avec l'oreillette droite. Une excision chirurgicale de la masse sous circulation extracorporelle a pu être réalisée. L'examen pathologique de la masse a confirmé la présence d'une importante colonie fongique de l'espèce Candida dans le thrombus. Les signes et symptômes d'obstruction de la veine cave supérieure ont disparu, et le patient a reçu son congé de l'hôpital quatre semaines plus tard, dans un état stable.

Conclusion: Bien que généralement causé par une compression tumorale extrinsèque, le syndrome de la veine cave supérieure peut être provoqué par une obstruction intravasculaire de la veine cave. Cette étiologie devrait faire partie du diagnostic différentiel, tout particulièrement chez les patients dotés d'appareils intravasculaires. L'échocardiographie transœsophagienne constitue un outil diagnostic précieux dans de tels cas.

$\mathrm{T}$ he constellation of signs and symptoms that results from obstruction of the superior vena cava (SVC) is known as SVC syndrome. This syndrome is most commonly caused by extrinsic caval compression produced by malignant mediastinal tumours. In recent years, increased use of intravascular devices such as catheters and pacemakers has exposed patients to the attendant risks, such as thrombosis and vascular strictures. As a result, SVC syndrome caused by nonmalignant conditions is being encountered wi th increasing frequency. Here we report a case of SVC syndrome caused by a large infected right atrial (RA)-SVC junction thrombus on a dialysis catheter. This condition was diagnosed using transesophageal echocardiography and was success-

From the Departments of Anesthesiology* and Surgery, $\uparrow$ Duke University Medical Center, Veterans Affairs Medical Center, Durham, North Carolina, USA.

Address correspondence to: Dr. Atilio Barbeito, Department of Anesthesiology, Box 3094, Duke University Medical Center, Durham, NC

27710, USA. Phone: 919-286-6938; Fax 919-286-6853; E-mail: barbe030@mc.duke.edu

Conflicts of interest: None declared.

The work presented in this manuscript was performed at the Veterans Affairs Medical Center, Durham, North Carolina.

Accepted for publication January 17, 2008.

Revision accepted August 25, 2008. 


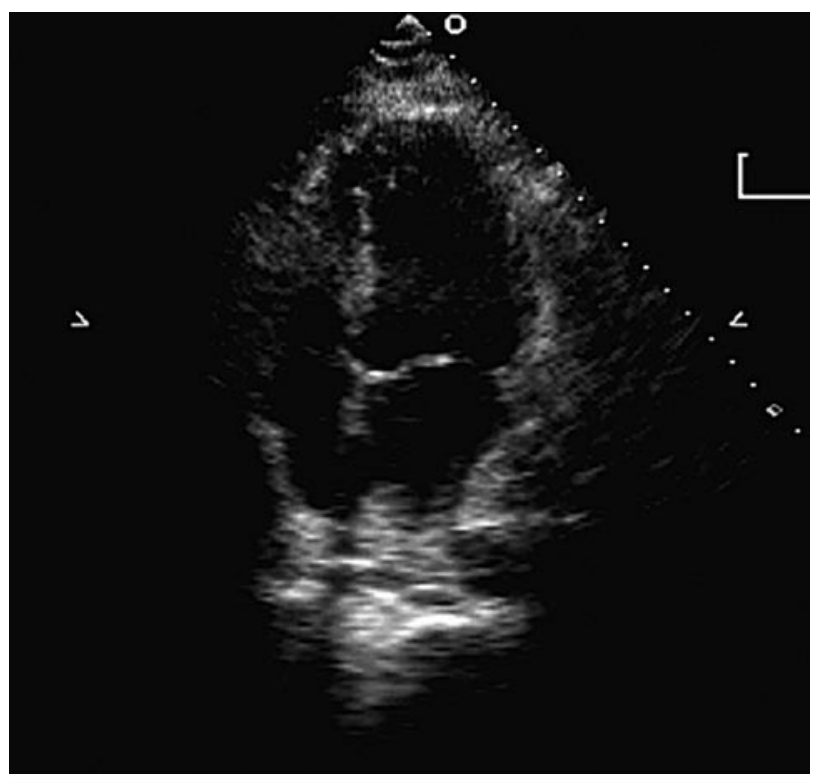

FIGURE 1 Transthoracic apical four chamber echocardiographic view of the heart. No evidence of endocarditis is seen.

fully treated with surgical intervention. The case highlights the need to consider the nonmalignant causes of SVC syndrome, the diagnostic role of transesophageal echocardiography, and the perioperative management of such patients. Written informed consent for the publication of images was obtained from this patient.

\section{Case report}

A 59-yr-old male was admitted to the hospital for treatment of a fungal infection. He had a history of type 2 diabetes mellitus complicated by retinopathy, neuropathy, and nephropathy requiring hemodialysis via a percutaneous tunnelled catheter. Prior to hospital admission, he had received a three-month course of amoxicillin for a dental infection. Five days prior to admission, he developed fever and chills during hemodialysis. Blood cultures obtained during this episode eventually demonstrated yeast. Based on this information, the patient was hospitalized for treatment of fungemia. Upon admission to the hospital, the tunnelled dialysis catheter was removed, and antifungal therapy was initiated with fluconazole. Shortly after admission, the patient was transferred to the intensive care unit because of altered mental status and hypothermia. His mental status improved over the ensuing few days, but blood cultures continued to show fungemia, Candida tropicalis, growing despite continued antifungal therapy. During this period of hospitalization, the patient also developed facial and bilateral upper extremity edema. A transthoracic echo-

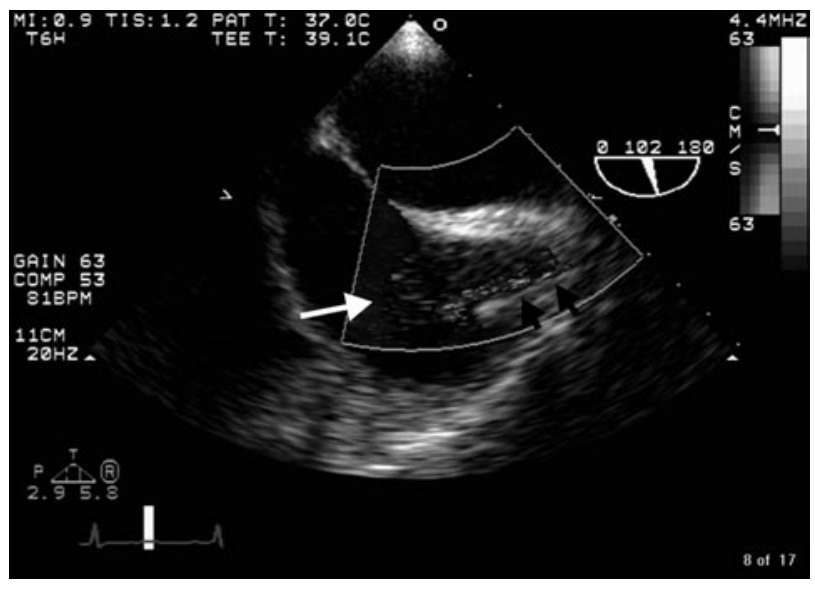

FIGURE 2 Transesophageal midesophageal bicaval echocardiographic view of the right atrium showing an echogenic mass at the right atrial-superior vena caval junction (white arrow). Colour Doppler imaging shows turbulent flow at the level of the obstruction (black arrows).

cardiogram (TTE) showed no evidence of endocarditis (Figure 1), but a transesophageal echocardiogram (TEE; Sonos 7500, Philips Medical Systems, Bothell, WA, USA) revealed a $2.5 \times 1.4 \mathrm{~cm}$ mass at the RASVC junction causing partial occlusion of the SVC and turbulent RA inflow (Figure 2), which had not been detected using TTE. There were no cardiac valvular vegetations seen on the TEE examination. Anticoagulation therapy was initiated, and antifungal coverage was broadened with amphotericin B. The patient developed recurrent fevers, progressive dyspnea, and worsening facial and upper extremity edema over the next eight days. A chest computerized tomography scan confirmed the presence of a filling defect in the RA extending into the SVC and excluded extrinsic caval compression. It also revealed bilateral pulmonary nodular opacities concerning septic emboli.

The patient was scheduled for RA thrombectomy using cardiopulmonary bypass. A careful examination of the patient's airway revealed no significant upper airway edema that would preclude intubation using direct laryngoscopy. General anesthesia was induced uneventfully, and intravenous access in the lower extremities was obtained. Blood pressure was maintained close to pre-induction values during the pre-bypass period.

Intraoperative TEE confirmed the presence of a large echo-dense mass at the SVC-RA junction, which had increased in size since the previous TEE examination. With cardiopulmonary bypass support, the patient underwent surgical excision of a $2.6 \times 1.4$ $\mathrm{cm}$ yellow-tan tubular mass from the RA-SVC junc- 


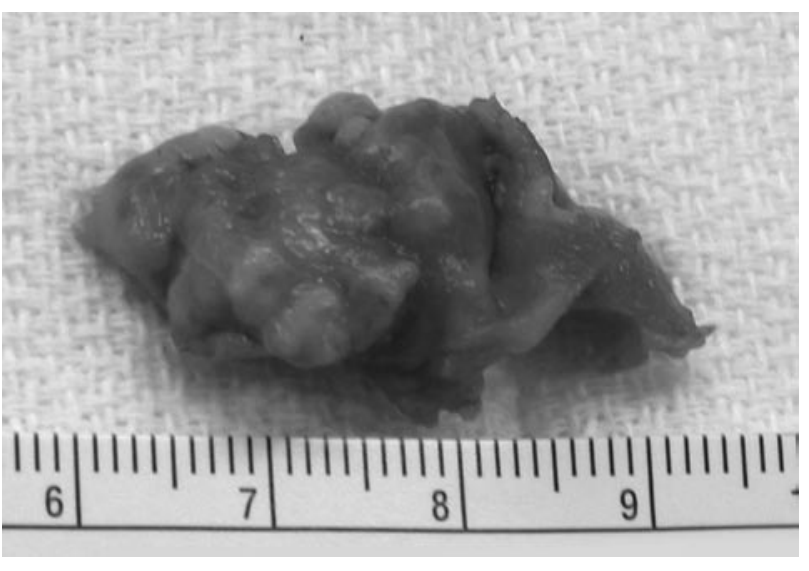

FIGURE 3 Right atrial thrombus.

tion. Pathological examination of the mass (Figure 2) confirmed the presence of a large fungal colony with morphology consistent with a Candida species mixed in the thrombus.

The patient's signs and symptoms of SVC obstruction resolved, but his postoperative course was initially complicated by hemorrhage requiring transfusion of blood products, and then delirium and atrial fibrillation, both of which resolved over the ensuing days. He was discharged from the hospital four weeks later in stable condition.

\section{Discussion}

Superior vena cava syndrome refers to the constellation of signs and symptoms resulting from obstruction of SVC flow. When the obstruction to flow is slow and progressive, decompressive collateral pathways develop, and symptoms are usually mild or absent. On the contrary, rapidly progressive obstruction of the SVC can lead to edema of the face, neck, and upper extremities, distention of collateral veins, dyspnea, and signs of increased intracranial pressure.

Although it is most frequently caused by extrinsic compression by tumours, SVC syndrome caused by nonmalignant conditions is increasing in frequency, likely reflecting the increased use of intravascular devices such as pacemakers, implantable cardioverterdefibrillators, and long-term indwelling venous catheters. ${ }^{1}$ Among the malignant causes of SVC syndrome are primary bronchial carcinomas, lymphomas, and other mediastinal neoplasms such as malignant thymomas and teratomas. The two most frequent causes of benign SVC syndrome are fibrosing mediastinitis and catheter-related thrombosis or vein stenosis. ${ }^{2}$ Benign etiologies have classically been reported to have an incidence of up to $22 \%$, but now comprise up to $40 \%$ of cases. ${ }^{3,4}$

Although thrombus formation is a relatively common complication of indwelling central venous catheters and pacemakers, ${ }^{5,6}$ the development of symptomatic SVC syndrome is rare, with a reported incidence of 0.03 to $0.04 \%{ }^{7,8}$ Superior vena cava syndrome caused by thrombus with a superimposed fungal infection is also rare. Of note, removal of the catheter does not eliminate the risk of obstruction, and SVC syndrome has been previously reported to develop after catheter removal, as in our case. ${ }^{9}$

In this case, catheter tip location in the RA may have been a predisposing factor for thrombus formation, owing to catheter irritation of the RA endocardium. ${ }^{10,11}$ In addition, our patient presented several risk factors for fungemia, including diabetes mellitus, renal failure, hemodialysis, and previous antibiotic exposure. ${ }^{12}$ The most common complication of fungal endocarditis is embolism (as seen in our patient), although congestive heart failure, sepsis, and other complications have also been reported. ${ }^{12}$

Since the patient's principal diagnosis was endocarditis, an echocardiogram was the first imaging study that was obtained. Transthoracic echocardiography is an excellent initial technique to evaluate and diagnose cardiac masses ${ }^{13}$ although the superior sensitivity of TEE for detecting vegetations has now been well established. ${ }^{14}$ In part, TEE transducers operate at higher ultrasonic frequencies, thereby improving image quality and spatial resolution. Additionally, the proximity of the esophagus and the heart eliminates ultrasound interference from interposed tissues. Using the modified Duke criteria, ${ }^{14}$ TEE should be the diagnostic test of choice in patients with at least "possible infective endocarditis" and a negative TTE examination.

In our case, a negative TTE evaluation and an intermediate pretest probability for infective endocarditis warranted further evaluation. Using TEE, we confirmed the diagnosis of endocarditis and SVC syndrome, and thereby aided the appropriate medical and definitive surgical treatment for this patient.

Computed tomography or magnetic resonance with gadolinium contrast are generally most useful in patients presenting with SVC syndrome. ${ }^{2}$ These studies have the added advantage of detecting mediastinal masses, which remain the most frequent cause of this syndrome. Once the gold standard diagnostic study, venography is now reserved for defining the anatomy preoperatively and for confirming vessel patency postoperatively in patients with SVC obstruction. ${ }^{4}$

Thrombosis of the SVC can be treated conserva- 
tively with anticoagulation, or with thrombolysis, percutaneous angioplasty (with or without stenting), or surgery. ${ }^{15-17}$ Although anticoagulation was initially attempted in this patient, the development of symptoms of SVC obstruction, the septic nature of the thrombus, and the development of embolic complications prompted the surgical treatment.

Most descriptions of the anesthetic management of patients with SVC syndrome include considerations for anterior mediastinal mass, which is often present in patients coming to the operating room with SVC obstruction. ${ }^{18}$ In these cases, the compressive effects of the mediastinal mass on the airway and major vascular structures provide the predominant anesthetic challenges. Upon induction of anesthesia, airway compression can cause complete airway collapse and loss of muscle tone, even in asymptomatic patients. Maintenance of spontaneous ventilation, together with induction of anesthesia in the semi-upright position, is often favoured. Compression of the SVC by the mediastinal mass also reduces preload and cardiac output, and, therefore, preload augmentation should be considered prior to induction of general anesthesia. In some cases, compression of the heart by the mass or a concurrent pericardial effusion can exacerbate these hemodynamic problems, and induction with ketamine or etomidate may be preferred in order to avoid the negative inotropic effects of other agents.

In this case, no external compression of the airway, major vessels, or the heart existed. The only physiologic derangement present was a limitation to venous inflow from the upper body caused by intravascular obstruction. Preload augmentation and a careful airway assessment to exclude significant airway edema were sufficient to safely anesthetize this patient. Intravenous access in the lower extremities is also recommended in these situations, in case blood return from the upper body becomes completely interrupted. If significant, obstruction to venous drainage of the upper body may compromise cerebral perfusion pressure. To avoid this, arterial blood pressure should be maintained as close as possible to pre-induction levels. The use of regional cerebral oximetry should be considered, as this monitoring modality has been shown to detect cerebral insults promptly when changes in arteriovenous pressure difference compromise brain perfusion. ${ }^{19}$ The reduction in cerebral metabolic rate caused by inhaled anesthetic agents, but not nitrous oxide, is an additional protective effect.

In conclusion, this case highlights a rare cause of SVC syndrome resulting from an infected RA-SVC thrombus and points out the improved sensitivity of TEE, compared to chest wall echocardiography, for making the diagnosis. Although this complication appears to be rare, it is important that it be recognized, since it is a potentially curable cause of SVC syndrome. Anesthetic management of patients with SVC syndrome may be challenging. The clinician needs to consider the etiology of the obstruction, as well as the possibility for compression of the airway, major vessels, and heart when a mediastinal mass is also present.

\section{References}

1 Wilson LD, Detterbeck FC, Yabalom J. Clinical practice. Superior vena cava syndrome with malignant causes. N Engl J Med 2007; 356: 1862-9.

2 Seelig MH, Oldenburg WA, Klingler PJ, Odell JA. Superior vena cava syndrome caused by chronic hemodialysis catheters: autologous reconstruction with a pericardial tube graft. J Vasc Surg 1998; 28: 556-60.

3 Kogon BE, Plattner C, Jennings S, Lyle T, McConnell M, Book WM. Cyanosis produced by superior vena caval stenosis. Ann Thorac Surg 2008; 85: 1083-5.

4 Rizvi AZ, Kalra M, Bjarnason H, Bower TC, Schleck $C$, Gloviczki P. Benign superior vena cava syndrome: stenting is now the first line of treatment. J Vasc Surg 2008; 47: 372-80.

5 Ghani MK, Boccalandro F, Denktas AE, Barasch E. Right atrial thrombus formation associated with central venous catheters utilization in hemodialysis patients. Intensive Care Med 2003; 29: 1829-32.

6 Spittell PC, Hayes DL. Venous complications after insertion of a transvenous pacemaker. Mayo Clin Proc 1992; 67: 258-65.

7 Chamorro H, Rao G, Wholey $M H$. Superior vena cava syndrome: a complication of transvenous pacemaker implantation. Radiology 1978; 126: 377-8.

8 Goudevenos JA, Reid PG, Adams PC, Holden MP, Williams DO. Pacemaker-induced superior vena cava syndrome: report of four cases and review of the literature. Pacing Clin Electrophysiol 1989; 12: 1890-5.

9 Al-Hilali N, Nampoory MR, Ninan VT, et al. The superior vena cava syndrome: late presentation after hemodialysis catheter removal. Saudi J Kidney Dis Transpl 2003; 14: 186-9.

10 Gilon D, Schechter D, Rein AJ, et al. Right atrial thrombi are related to indwelling central venous catheter position: insights into time course and possible mechanism of formation. Am Heart J 1998; 135 : 457-62.

11 Fuchs S, Pollak A, Gilon D. Central venous catheter mechanical irritation of the right atrial free wall: a cause for thrombus formation. Cardiology 1999; 91: 169-72. 
12 Pierrotti LC, Baddour LM. Fungal endocarditis, 19952000. Chest 2002; 122: 302-10.

13 Ragland MM, Tak T. The role of echocardiography in diagnosing space-occupying lesions of the heart. Clin Med Res 2006; 4: 22-32.

14 Krivokapich J, Child JS. Role of transthoracic and transesophageal echocardiography in diagnosis and management of infective endocarditis. Cardiol Clin 1996; 14: 363-82.

15 Dreyer G, Bingham C. Right atrial thrombus as a complication of a temporary haemodialysis catheter-a potentially avoidable complication. Nephrol Dial Transplant 2005; 20: 474-5.

16 Fasano R, Kent P, Valentino L. Superior vena cava thrombus treated with low-dose, peripherally administered recombinant tissue plasminogen activator in a child: case report and review of the literature. J Pediatr Hematol Oncol 2005; 27: 692-5.

17 Akoglu H, Yilmaz R, Peynircioglu B, et al. A rare complication of hemodialysis catheters: superior vena cava syndrome. Hemodial Int 2007; 11: 385-91.

18 Bechard P, Letournean L, Lacasse $\Upsilon$, Cote D, Bussieres $J S$. Perioperative cardiorespiratory complications in adults with mediastinal mass: incidence and risk factors. Anesthesiology 2004; 100: 826-34; discussion 5A.

19 Reece IJ, al Tareif H. Surgical interruption of the superior vena cava. J Thorac Cardiovasc Surg 1995; 109: 1020 . 